\title{
On the motion of small spheroidal particles in a viscous liquid
}

\author{
By P. G. SAFFMAN \\ Trinity College, Cambridge
}

(Received 22 May 1956)

\begin{abstract}
SUMmary
Small spheroidal particles suspended in a sheared viscous liquid are sometimes observed to take up slowly preferred orientations, relative to the motion of the undisturbed liquid, which are independent of the initial conditions of release. These observations cannot be accounted for by the solution, obtained by Jeffery (1922), of the linearized Navier-Stokes equations. It is shown in this paper that the effect of the inertia of the liquid is to alter slowly the orbit of the particle in accordance with Jeffery's hypothesis that the particle ultimately moves in such a way that the dissipation of energy is a minimum, but that this effect is orders of magnitude too small to account for any of the experimental observations.

It is suggested that non-Newtonian properties of the liquid account for the observations. It is shown that the rate of orientation of a particle would then be independent of its size, and this prediction is verified experimentally. Other experimental evidence in support of this suggestion is also described.

Some remarks are also made about the possible effect of collisions between the particles when more than one particle is present.
\end{abstract}

\section{INTRODUCTION}

'The slow motion of a small ellipsoidal particle in a uniformly sheared viscous fluid was examined by Jeffery (1922). The usual approximation was made that the acceleration (commonly called inertia) terms $\rho(\partial \mathbf{u} / \partial t+\mathbf{u} . \nabla \mathbf{u})$ in the hydrodynamical equations of motion may be neglected when the Reynolds number is sufficiently small. The approximate equations of motion

$$
\mu \nabla^{2} \mathbf{u}-\nabla p=0, \quad \operatorname{div} \mathbf{u}=0,
$$

were then solved with the boundary condition that $\mathbf{u}=\boldsymbol{\Omega} \wedge \mathbf{r}$ at the surface of the ellipsoid. Here, $\mathbf{u}, p, \mu$ and $\rho$ denote respectively the velocity, pressure, viscosity and density of the liquid, $\boldsymbol{\Omega}$ is the angular velocity of the ellipsoid (to be found) and the origin of $\boldsymbol{r}$ is the centre of the ellipsoid. The angular velocity was determined by the condition that the total force and 
couple on the body should vanish; this condition is valid since, as can be shown, the inertia of the body may be neglected to the same order in the Reynolds number as the approximation involved in (1).

The equations that determine the motion or orbit of the ellipsoid can be integrated when the ellipsoid is one of revolution. Let $\theta$ be the angle between the axis of symmetry of the spheroid and the direction of the vortex lines of the undisturbed shear, and let $\phi$ be the angle between the plane that contains the axis of symmetry and the direction of the vortex lines and a fixed plane containing the latter direction, that is, $\phi$ is the azimuthal angle of the axis of symmetry about the direction of the vortex lines. Then the orbit of the spheroid is given by the equations

$$
\left.\begin{array}{rl}
\left(a^{2}+b^{2}\right) \dot{\theta} & =\kappa\left(a^{2}-b^{2}\right) \sin \theta \cos \theta \sin \phi \cos \phi, \\
\left(a^{2}+b^{2}\right) \dot{\phi} & =\kappa\left(a^{2} \cos ^{2} \phi+b^{2} \sin ^{2} \phi\right),
\end{array}\right\}
$$

which have the solution

$$
\left.\begin{array}{l}
\tan \phi=\frac{a}{b} \tan \frac{\kappa a b t}{a^{2}+b^{2}}, \\
\tan \theta=\frac{a b}{k\left(a^{2} \cos ^{2} \phi+b^{2} \sin ^{2} \phi\right)^{1 / 2}},
\end{array}\right\}
$$

where $2 a$ is the length of the axis of symmetry, $2 b$ is the length of the other principal axes, $\kappa$ is the magnitude of the undisturbed shear, and $k$, the orbital parameter, is an arbitrary constant of integration that depends upon the initial conditions of release.

These equations show that the motion is periodic with the axis of symmetry describing a (non-circular) cone about the direction of the vortex lines, so that the motion is kinematically similar to that of a top. It also follows that the value of $k$, which specifies the particular orbit, is determined by the initial conditions, and there is no tendency for the spheroid to set itself so that its axes point in a particular direction or to choose a particular orbit, that is, there are no preferred orientations relative to the motion of the surrounding fluid that the spheroid will eventually take up irrespective of the initial conditions.

Jeffery did not consider the existence of this indeterminateness to be entirely satisfactory and expressed the opinion that a more complete investigation would reveal the existence of preferred orbits. In effect, the suggestion made was that over a short period of time the orbit is given accurately by equations (2) and (3), but that effects neglected in the analysis have a cumulative effect and slowly alter the orbit, so that eventually the particle is moving in a preferred orbit which is independent of the initial conditions. Jeffery suggested further that these preferred orbits would correspond to those solutions of the approximate equations of motion (1) for which the rate at which energy is dissipated by viscosity is a minimum. It was then shown that, according to this hypothesis, prolate spheroidal particles would tend to set themselves with the axis of symmetry parallel to the vortex lines 
and would rotate about this axis with constant angular velocity, so that the whole motion is steady; and oblate spheroidal particles would tend to set themselves with an equatorial axis parallel to the vortex lines and would rotate about this axis with a variable angular velocity, so that the whole motion is periodic but not steady.

Several experiments have been performed in order to test the accuracy of equations (2) and (3) and to investigate whether preferred orbits do indeed exist. The results of these experiments, which will be described briefly in $\S 2$, are not uniform. In some cases, preferred orbits do not appear to exist; in other cases, they do exist but are orbits of either minimum or maximum energy dissipation.

This question of preferred orbits is not without practical interest; for example, it is closely related to the viscosity of suspensions of non-spherical particles. It is the purpose of this paper to discuss some of the effects neglected by Jeffery in order to see whether there are any theoretical reasons why particles should take up preferred orbits in accordance with Jeffrey's hypothesis, and to suggest a possible explanation of the experimental results.

In $\$ 3$ we shall discuss the problem generally and show that many effects, such as those due to the inertia of the particle or the presence of rigid walls, need not be considered; and that the two most likely effects are the inertia of the liquid, which gives rise to the acceleration terms in the equations of motion, and non-Newtonian properties of the liquid. The rate at which the orbit of the particle is altered by the inertia of the liquid was calculated and the results are given in $\S 4$. These results provide theoretical support for Jeffery's hypothesis, but are unable to account for any of the experimental observations since the predicted rates of orientation are orders of magnitude too small. It is suggested in $\S 5$ that non-Newtonian properties of the liquid are responsible for the observed phenomena, and experimental evidence in support of this suggestion is described.

When more than one particle is present, as is the case for a suspension, there is a further effect to be considered, namely that of collisions and interactions between the particles (see Manley, Arlov \& Mason 1955). In $\S 6$, we shall give the results (mainly negative) of calculating the mechanics of the interaction between two particles whose separation is always large compared with their size, and discuss briefly the significance of these results and some intuitive ideas about the problem.

\section{RELEVANT EXPERIMENTAL RESULTS}

Experiments using prolate and oblate spheroids were carried out by Taylor (1923). Binder (1939), Trevelyan \& Mason (1951), and Manley \& Mason (1956) describe experiments in which cylindrical rods of large aspect ratio were used (the aspect ratio is the ratio of the length to the diameter). In all these experiments, the particles were suspended in liquids contained between rotating concentric cylinders. This motion differs from the uniform shear of infinite extent envisaged by Jeffrey in the 
existence of Coriolis forces, wall and end effects, and the fact that the shear in the neighbourhood of the particle is only approximately uniform. However, these effects can easily be shown to be negligible to the order considered by Jeffery, although their possible influence on the existence of preferred orbits has to be kept in mind (see $\$ \S 3$ and 4 below).

Taylor used aluminium spheroids in water-glass. He observed that, as far as he could tell, the spheroids moved as predicted by equations (2) and (3), and that, after about 20 minutes for the prolate spheroids and five minutes for the oblate ones (these figures correspond roughly to $180 \mathrm{com}$ plete revolutions for the prolate spheroids and 40 for the oblate ones), the spheroids took up orbits in accordance with Jeffery's hypothesis. In these experiments, $\kappa \div 1.65 \mathrm{sec}^{-1}, \epsilon \doteqdot 0.5$ when $a \doteqdot 0.13 \mathrm{~cm}$, and $\epsilon \div-0.75$ when $a \doteqdot 0.05 \mathrm{~cm}$. Here $\epsilon$ denotes the ratio $(a-b) / a$, sometimes called the 'ellipticity' of the spheroid. 'The viscosity of water-glass is very roughly $1,000 \mathrm{gm} / \mathrm{cm} \mathrm{sec}$.

Binder used particles of human hair suspended in glycerine. The orbits of the particles are described as being in qualitative agreement with equations (2) and (3), although there must be some discrepancy since these equations describe the motion of spheroids and not cylindrical rods. When the aspect ratio of the rods was less than 15 , the rods orientated themselves along the vortex lines, in accordance with Jeffery's hypothesis, but when the aspect ratio was greater than 15 , the rods set themselves perpendicular to the vortex lines in an orbit of maximum energy dissipation.

Trevelyan \& Mason and Manley \& Mason used cylindrical rods of glass, dacron, nylon and human hair in corn syrup. They verified equations (2) and (3) to a reasonable degree of accuracy considering that rods and not spheroids were used, but their observations of the existence of preferred orbits for a single particle are inconclusive. They sometines observed a slow tendency for a particle to align itself along the vortex lines or to take up an orbit in which it was perpendicular to the vortex lines, but in other cases the orbit varied erratically with time. The manner in which the orbit varied appeared to be random and independent of the particle size, the aspect ratio, and the magnitude of the undisturbed shear.

Manley \& Mason carried out also experiments with suspensions of particles of large aspect ratio. Suspensions in which the particles were initially distributed isotropically were sheared, and a statistical tendency was found for those orbits with large values of $k$ to be favoured, that is, there was a tendency for particles to orientate themselves along the vortex lines.

\section{ThE NON-LINEARITY OF THE PROBLEM}

We shall now consider whether a theoretical treatment which uses the approximate equations (1) can explain these experimental observations or predict the existence of preferred orbits. In other words, when the perturbations due to the effect of rigid walls in the experimental apparatus, or the possibility that the shear is only approximately uniform, or any similar 
alterations in the idealized external conditions envisaged by Jeffery, are taken into account using the approximate equations (1), does the orbit of the particle remain constant in time or alter slowly until a preferred orbit is reached?

Now equations (1) are linear and hence all pressures and stresses are proportional to the magnitude of the undisturbed shear and are therefore linear in $\kappa$. It follows that the equations determining the orbit are linear in $\kappa$, i.e. $\dot{\theta}$ and $\dot{\phi}$ are given by the products of $\kappa$ and functions of $\theta$ and $\phi$. The extra effects can be regarded as giving rise to perturbations of the orbit given by (3), and any variations of the orbit with time are then best represented by changes in the mean value of the orbital parameter $k$, where $k$ can vary between 0 and $\infty$, being zero when the axis of symmetry is perpendicular to, and infinite when parallel with, the vortex lines, and the mean is taken over a complete revolution of the particle. On putting $\theta$, as given by (3), in the expressions obtained for $\dot{\theta}$ and $\dot{\phi}$ when the extra effects are taken into account, and averaging over a complete revolution, we then obtain

$$
\frac{1}{k} \frac{d k}{d t}=\kappa f(k)
$$

where $f(k)$ depends on the shape but not the size of the particle and (as in all subsequent equations of this kind) $k$ is to be understood as representing the average value over a complete revolution. Hence, if the shear is reversed, by, for example, the cylinders being rotated in opposite directions, then $d k / d t$ will change sign; and if a preferred orbit exists in one case, a different preferred orbit will arise when the shear is reversed.

It follows immediately that, if the motion is such that the physical situation is unaltered by a reversal of the shear, then $f(k)$ must be zero and the particle rotates in a constant orbit. In other words, the use of equations (1) will predict that the orbit remains fixed unless there is a certain degree of asymmetry in the external conditions. (Another way of seeing this result is to note that, according to equations (1), the motion is reversible.) This result was indeed verified directly for the case of a spheroid near a rigid plane wall. The wall effect was calculated by a method due to Lorentz (1907), and the necessary modifications to (2) and (3) were found. It was found, as expected, that the mean value of $k$ over one revolution remains constant.

Further, this argument also shows that the inertia of the particle may be neglected since the equations for the orbit, with the inertia of the particle taken into account, are linear in $\kappa$.

It is clear now that the question of preferred orbits is to some extent dependent on the design of the experimental apparatus. However, the Couette flow between rotating cylinders that was used in the experiments described in the previous section and with which we are primarily concerned possesses the necessary symmetry for the above result to be valid. Further, it is to be expected that flows for which the symmetry condition is not valid will be the exception rather than the rule; and even in these cases, the result 
may still hold since the linearity of equations (1) and the consequent superposability of solutions enables the motion to be split into two parts, one of which is reversible and the other not, and the part that is not reversible may not produce a couple on the particle. For example, if account is taken of the relative velocity of particle and liquid owing to their different densities, this relative velocity does not change sign when the shear is reversed but the couple it produces on the particle is zero.

We must therefore look for non-linear terms that are neglected in (1). The first such terms that come to mind are the acceleration (inertia) terms and in the next section we shall examine their effect. We shall see, however, that the experimental phenomena cannot be explained in this way and some other non-linear effect must be sought. Non-Newtonian properties of the liquid give rise to non-linear effects and these will be discussed in $\S 5$ together with some relevant experimental results.

There is, of course, the possibility that external effects of a random nature influence the orbits of the particles. It is difficult to understand the experiments with single particles by Manley \& Mason (1956) in any other way. Indeed, they say that slow convection currents were present and regard these as the explanation of the randomness of their observations, and the opinion is expressed that the orbit would remain constant in the absence of such random effects. However, experiments that are capable of being repeated, such as those of Taylor or those described in $\S 5$, cannot be explained in this way.

\section{The INERTIA OF THE LIQUid}

The full equations of motion of an incompressible liquid are

$$
\rho \frac{D \mathbf{u}}{D t}=-\nabla p+\mu \nabla^{2} \mathbf{u}, \quad \operatorname{div} \mathbf{u}=0 .
$$

To calculate the effect of the acceleration terms by obtaining the complete solution of (5) is, in general, a problem of great difficulty, but we can by simple dimensional considerations obtain the order of magnitude of their effect. For $\rho(D \mathbf{u} / D t) / \mu \nabla^{2} \mathbf{u}$ is of order $R$, where $R$ denotes the Reynolds number $\kappa a^{2} / \nu(\nu \equiv \mu / \rho)$, and on making the plausible assumption that the extra stresses introduced when the acceleration terms are taken into account are smaller by a factor of order $R$ than the stresses calculated neglecting these terms, we obtain for the rate of change of the orbit due to the inertia of the liquid

$$
\frac{1}{k} \frac{d k}{d t}=\frac{a^{2} \kappa^{2} \epsilon}{\nu} g\left(\frac{k}{a}\right)
$$

where $g(k / a)$ depends on the shape of the particle, and the factor $\epsilon(=(a-b) / a)$ has been introduced to emphasize the fact that $d k / d t$ vanishes from considerations of symmetry when the particle is a sphere.

Now the sign of $g(k / a)$ is of interest in connection with Jeffery's hypothesis, and it is also not without value to have an estimate of its numerical magnitude. It was therefore decided to calculate this function by taking 
account of the acceleration terms in (5) by means of an iterative procedure. That is, denoting the solution of (5) with $\rho D \mathbf{u} / D t$ neglected by $\mathbf{u}^{(1)}$ and $p^{(1)}$, where $\mathbf{u}^{(1)}=\boldsymbol{\Omega} \wedge \mathbf{r}$ at the surface of the spheroid and $\mathbf{u}^{(1)}$ tends to the undisturbed velocity as $r \rightarrow \infty$ (this solution is, of course, the one found by Jeffery), we consider the equations

$$
\mu \nabla^{\mathbf{2}} \mathbf{u}-\nabla p=\rho \frac{D \mathbf{u}^{(1)}}{D t}, \quad \operatorname{div} \mathbf{u}=0,
$$

and try to find a solution which satisfies the boundary conditions $\mathbf{u}=\boldsymbol{\Omega} \wedge \mathbf{r}$ on the spheroid and $\mathbf{u}-\mathbf{u}^{(1)} \rightarrow 0$ as $r \rightarrow \infty$.

A most serious difficulty now arises since it can be shown that no solution of $\left(5^{\prime}\right)$ can be constructed which satisfies the boundary condition at infinity: this is sometimes known as Whitehead's paradox and was first encountered by Whitehead (1893) when considering the uniform translation of a sphere. However, there are reasons for believing that this difficulty can be overcome in the following way: there is evidence that if a suitable boundary condition at infinity is chosen for $\mathbf{u}$, then the solution of $\left(5^{\prime}\right)$ that satisfies this boundary condition will represent accurately in the neighbourhood of the particle the second approximation to the solution of (5). This boundary condition at infinity is deduced from the solution of the Oseen-type equations for the problem, but in the present problem these equations are difficult and have so far not yet been solved. However, by analogy with simpler cases, it appears that the requisite boundary condition is $\left|\mathbf{u}-\mathbf{u}^{(1)}-\lambda R \mathbf{u}^{(1)}\right|=o(r)$ as $r \rightarrow \infty$, where $\lambda$ is a numerical constant (possibly zero) of order one. Equations $\left(5^{\prime}\right)$ can then be solved, the second approximation to the couple on the particle can be calculated in terms of $\Omega, \kappa$ and $\lambda$, and the orbit of the particle can be determined. It is clear that the effect of $\lambda$ is equivalent to a small change in the magnitude of the undisturbed shear and so, to the order considered, $\lambda$ will not enter the expression for $d k / d t$ and its actual value is not needed for the purpose of this paper. It must be emphasized, however, that this boundary condition may well be wrong and this doubt is to be kept in mind whilst interpreting the results obtained below.*

\section{Result of the calculation}

Although it seems possible, in principle, to carry out the iteration for a spheroid of arbitrary shape, the analysis that appears to be required is such that no practical method could be found. The analysis was therefore confined to the comparatively simple case of a nearly spherical spheroid, so that powers of $\epsilon$ higher than the first could be neglected. The solution was then obtained by expanding the velocity and pressure in series of solid harmonics multiplied by powers of $r$. Since the algebra is tedious and

\footnotetext{
* I am deeply indebted to Dr I. Proudman for information and advice concerning the validity of iterated solutions of the Navier-Stokes equations and about the boundary condition to be satisfied at infinity. It is regretted that a description of the reasons for suggesting the above boundary condition would be quite lengthy and out of place in the present context.
} 
involved, it would serve no useful purpose to reproduce it here and only the final result will be given.

It was found that when the acceleration terms are taken account of in the above manner,

$$
\frac{1}{k} \frac{d k}{d t_{i}} \div 0.24 \frac{a^{2} \kappa^{2} \epsilon}{\nu}
$$

This result is equivalent to $g(k / a)$ having the value $0 \cdot 24+O(\epsilon)$.

For a prolate spheroid, $\epsilon>0$ and so $d k / d t>0$, i.e. the long axis tends to set itself in the direction of the vortex lines; for an oblate spheroid, $\epsilon<0$ and so $d k / d t>0$, i.e. the short axis tends to set itself perpendicular to the vortex lines. Thus, the effect of the inertia of the liquid is in accordance with Jeffery's hypothesis and provides theoretical support for this hypothesis.

\section{Comparison with experiment}

Let us now compare the observed rates of orientation with those predicted by equations (6) or (7). The relevant experiments are those of Taylor (1923) (these experiments were repeated by myself and similar results obtained). It is true that $\epsilon$ is not sufficiently small for (7) to be accurate, and that Coriolis forces of the same order of magnitude as the acceleration terms should be taken into account, but (7) should give the order of magnitude of the effect of the inertia of the liquid without serious error. (The ratio of Coriolis to acceleration terms is $\Omega^{\prime} / \kappa$ or $\left(\Omega^{\prime} / \kappa\right)^{2}$, according as to which is the larger, where $\Omega^{\prime}$ is the angular velocity of the particle around the axis of the cylinders; this ratio is either of order one or zero in the experiments described in this paper.) On putting the data given by Taylor into equation (7), or using equation (6) with the plausible assumption that $g(k / a)$ is of order one, we find that $(1 / k)(d k / d t)$ is at most $10^{-5} \mathrm{sec}^{-1}$. Now a change in the orbit should be observable when $k$ changes by a factor $e$, and the time for this to happen is not less than $10^{5}$ seconds $\div 28$ hours! Since the observed times were not greater than 20 minutes, there can be little doubt that the effects of the inertia of the fluid are too small to produce the observed phenomena.

When the aspect ratio of the particles is large, equation (7) is likely to be in serious error, and a direct comparison with the experimental observations on cylindrical rods cannot be made. However, a general examination of the equations for the case of large aspect ratio shows that $(1 / k)(d k / d t)$ is then probably proportional to $b^{2} \kappa^{2}(\log a / b)^{2} / \nu$. If this be so, it is easily shown that the times needed for a change of orbit to be clearly observable are orders of magnitude larger than those observed by Binder (1939), and are also long compared with the duration of the other experiments on cylindrical rods quoted previously.

\section{The motion of small particles in a tube}

Of further interest is another phenomenon predicted by Jeffery. It can easily be shown that small particles suspended in the liquid flowing 
through a capillary tube will, according to equation (1), remain at a fixed distance from the axis of the tube; however, it is a consequence of Jeffery's hypothesis of minimum energy dissipation that they should move slowly towards the axis since the shear there is least, so the extra energy dissipation owing to the presence of the particles is then reduced. (This phenomenon appears to be quite well known, and is observed, for example, by physiologists studying the motion of blood corpuscles in the capillaries of the body. I am not aware, however, of any accurate quantitative measurements that have been published.)

The effect of the inertia of the fluid on the motion of a small sphere of radius $a$ in a flow with a parabolic velocity profile $u=\alpha z+\beta z^{2}$ was worked out by iteration as before and it was found that the inertia terms give rise to a sideways velocity $w$, perpendicular to the directions of the flow and the vortex lines, given by

$$
w \doteqdot-0.43 \frac{\alpha \beta a^{4}}{\nu} .
$$

This velocity moves the sphere to a region where the magnitude of the shear is least, and so the effect of the inertia of the fluid is again in accordance with Jeffery's hypothesis. Dr M. J. Taylor has, however, communicated to me some rough experimental data of his own, from which it appears that the velocities predicted by (8) are too small by a factor of order 10 .

To sum up, if the inertia of the liquid is the dominant non-linear effect, then there is theoretical support for Jeffery's hypothesis. It appears, however, that in the experiments described in this paper, the inertia terms are too small to account for the observations, and we shall now go on to discuss the possible effects of non-Newtonian properties of the liquid.

\section{NON-SEWTONIAN PROPERTIES OF THE LIQUid}

It is assumed in the derivation of the Navier-Stokes equations (5) that the stress tensor $p_{i j}$ is linearly related to the rate-of-strain tensor

by the equation

$$
e_{i j}=\left(\frac{\partial u_{i}}{\partial x_{j}}+\frac{\partial u_{j}}{\partial x_{i}}\right)
$$

A liquid for which (9) does not hold is said to be non-Newtonian, and this definition obviously covers a wide range of liquids with various properties. However, we are concerned here with incompressible liquids which are almost Newtonian, that is, liquids in which anomalous effects caused by deviations from equation (9) are small. For such liquids, we may take the stress-strain relation as

$$
p_{i j}=-p \delta_{i j}+\mu e_{i j}+\sigma Q_{i j},
$$

in which $\mu$ is constant and $\sigma Q_{i j}$, where $\sigma$ is a constant with the dimensions of viscosity divided by time which is some measure of the non-Newtonian properties of the liquid, is a quadratic functional of the rate-of-strain tensor which may involve both the values of $e_{i j}$ at earlier times and higher spatial 
derivatives of $e_{i j}$. It is also supposed that the motion is sufficiently slow for powers of $e_{i j}$ higher than the second to be neglected.

The nature of the quadratic term in (10) is by no means clear. If it be supposed that the liquid is isotropic when at rest, that the stress depends only on the instantaneous rate-of-strain, and that the stress-strain relation is analytic, then it can be shown that $\sigma Q_{i j}$ may be taken as $\mu^{\prime} e_{i k} e_{k j}+\mu^{\prime \prime} e_{k l}^{2} \delta_{i j}$, where $\mu^{\prime}$ and $\mu^{\prime \prime}$ are constants (for a full review of the subject, see Truesdell (1952)). But in the present problem, where the motion is not steady, this form of the quadratic term may well not be valid. Apart from some evidence that $\mu^{\prime}$ is positive if this form of the quadratic term is valid, there appears to be no non-Newtonian liquid for which the form or magnitude of the quadratic term has been established.

The equations of motion of the liquid are $\partial p_{i j} / \partial x_{j}=0$, since the inertia of the liquid may be neglected. Because the non-Newtonian terms are supposed to be small, a solution of these equations could be attempted by iteration, provided that a suitable form for $Q_{i j}$ were postulated. (It is to be noted that the difficulty at infinity which arose when the acceleration terms were taken into account by iteration would not arise here.) It can be seen that, after solving the equations of motion and equating the couple on the particle to zero, we should finally obtain

$$
\frac{1}{k} \frac{d k}{d t}=\frac{\kappa^{2} \sigma}{\mu} h(k / a)
$$

where $h(k / a)$ depends on the shape of the particle and on the form of $Q_{i j}$. The right-hand side of (11) might be identically zero, but there is no a priori reason why this should be so and it seems most unlikely that this should be the case.

The rate of orientation of the particle is known when $h(k / a)$ is known but no attempt was made to determine $h(k / a)$ because the form of $Q_{i j}$ is not known beyond reasonable doubt. Even if $\sigma Q_{i j}$ were known or postulated with a reaso table degree of certainty, there appears to be no practical way of solving tl e equations for a spheroid of arbitrary shape, and to carry out the analysis for a nearly spherical spheroid would require very heavy algebra and would probably not be of much use since $\sigma Q_{i j}$ would probably contain sufficient unknown constants for agreement with experiment to be reached without difficulty. However, it follows from (11) that the rate of orientation of a particle of given shape in a given liquid is independent of its size; whereas the rate of orientation due to the inertia of the liquid is, for a given shape, proportional to the surface area of the particle. The following simple experiment was therefore carried out in order to see whether the rate of orientation is independent of the size; it was verified roughly that this is indeed the case.

\section{Experimental procedure}

The apparatus consisted of two concentric cylinders: the diameter of the outer one was $8.4 \mathrm{~cm}$ and that of the inner one was $2.8 \mathrm{~cm}$. Prolate and 
oblate spheroids, similar in shape but of different sizes were made on a lathe from aluminium wire. The longest and shortest axes of the smallest prolate spheroid were $0.25 \mathrm{~cm}$ and $0.10 \mathrm{~cm}$ respectively, and of the smallest oblate spheroid $0.17 \mathrm{~cm}$ and $0.10 \mathrm{~cm}$. The other spheroids were twice and three times as large. The outer cylinder rotated one revolution in 4.3 seconds and the inner one was kept at rest. The particles were placed midway between the cylinders so that $\kappa$ was about $0.82 \mathrm{sec}^{-1}$. Two liquids were used : a highly viscous form of ammonium polymethyl acrilate, and waterglass. The densities of these liquids were measured in the usual way and the viscosities by timing the rate of fall of a small lead sphere of known size. The density of the ammonium polymethyl acrilate was close to that of water, that of the water-glass was about 1.5 times that of water; the kinematic viscosities were, as it happened, approximately the same, being about $1000 \mathrm{~cm}^{2} \mathrm{sec}^{-1}$.

A common property of a non-Newtonian liquid is that, when confined between rotating cylinders, it will climb up the inner cylinder, provided the upper surface is free (see, for example, Weissenberg 1947). It was noticed that the ammonium polymethyl acrilate climbed up the inner cylinder by as much as one centimetre, whilst the water-glass did not climb up at all. This indicated that the non-Newtonian properties of the water glass were small compared with those of the other liquid.

The experimental results for the ammonium folymethyl acrilate were as follows. The prolate spheroids were placed with their long axis horizontal, and after about five minutes the long axis was vertical. The oblate spheroids were placed with their short axis vertical, and after about six minutes the short axis was horizontal. As far as I could tell from these crude observations, the rate of orientation was independent of the size of the particles. When water-glass was used, it was found that the orbits of the spheroids remained constant over times up to an hour.

These results are consistent with the suggestion that the explanation of the observed phenomena lies in non-Newtonian properties of the liquid. There is an apparent discrepancy, however, between these results for waterglass and the observations of Taylor (1923). But Sir Geoffrey Taylor has informed me that, in his experiment, the water-glass rose some distance up the inner cylinder, and this was also the case when I repeated Taylor's experiment with a different supply of water-glass and at probably a higher temperature than the experiments described above. Bearing in mind that water-glass is by no means a standard product, and that non-Newtonian properties are often critically dependent upon temperature (although the temperature dependence here is in the opposite direction from what would be intuitively expected), this apparent discrepancy appears to yield further evidence in favour of the above suggestion. Further, Dr S. G. Mason has informed me that the corn syrup used in the experiments in which he participated (where no preferred orbits were observed with single particles) was found to be Newtonian over a wide range of velocity gradients.

There thus seems to be strong evidence that the existence of preferred orbits is related to the effect of non-Newtonian viscosity and that some of 
the experimental observations of spheroidal particles suspended in the Couette flow between concentric cylinders will be explained when the action and effect of non-Newtonian properties can be calculated.

Further, $\sigma$ is a measure of the elastic properties of the liquid, since it is easily shown that the quadratic term in (10) does no work when the liquid is deformed and returned to its original state, so that $\sigma / \mu$ is a measure of the relative magnitudes of the elastic and viscous properties of the liquid.* ( $\sigma / \mu$ is probably proportional to $\mu / \gamma$, where $\gamma$ is the elastic modulus of rigidity of the liquid.) To account for the experiments described above, $\sigma / \mu$ for ammonium polymethyl acrilate would have to be of order $10^{-3} \mathrm{sec}^{-1}$.

An experiment to investigate the possible relationship between the elastic properties of a variety of non-Newtonian liquids and the rate at which preferred orbits are reached would be of great interest in this connection, as would also be an accurate experiment to check that the rate of orientation is proportional to the square of the undisturbed shear.

\section{Suspensions of MANY PaRTiCles}

When considering a suspension of many particles, we are primarily interested in the proportion of particles whose orbital parameters lie in a given range, since this is needed for a calculation of the bulk properties of the suspension and is also the quantity that is actually measured. That is, we wish to determine the values of the function $p(k)$ where $p(k) d k$ denotes the proportion of particles with orbital parameters in the range $(k, k+d k)$. If we were to follow a definite particle, we would find that its orbit is continually being altered in apparently discontinuous jumps by collisions with other particles (see Manley \& Mason 1956) and that there might also be a slow change due to the effects described in the previous sections. In this section, however, we shall not discuss these latter effects but confine ourselves to a discussion of the effect of collisions. (It is worth mentioning, in passing, that a suspension of non-spherical particles is anisotropic and this implies that the bulk properties of the suspension may be to some extent non-Newtonian. This in turn may effect the external motion-for example, a suspension contained between rotating cylinders may climb up the inner cylinder, even though the pure liquid does not-and so affect the motion of an individual particle and give rise to preferred orbits. This effect will not be discussed here.)

It does not necessarily follow, of course, that $p(k)$ is altered by collisions, for example, it may be that the effect of a collision is to interchange the orbital parameters of the particles that collide; however, there is no a priori evidence that this is the case and it seems most likely that the effect of collisions is to alter the orbits until a statistically steady state is reached. It might at first be thought that, if the interactions were calculated using equations (1), the linearity of the equations and the consequent reversibility of the motion would imply that $p(k)$ is not altered by collisions, since if

\footnotetext{
* 'This was pointed out to me by Dr K. Weissenberg.
} 
$d p(k) / d t$ is not zero, its sign is reversed by reversing the external shear. This is a well-known paradox of Statistical Mechanics, however, and it can in fact be shown that the reversibility of the motion does not imply that $d p(k) / d t=0$; see Tolman (1938) where this problem is discussed in connection with the Boltzmann $\mathrm{H}$-Theorem. In other words, it is sufficient to calculate the collisions using the approximate equations (1).

The possibility exists that the steady state could be calculated without a detailed knowledge of the mechanics of an interaction by means of the ideas of Statistical Mechanics. This can be done if it is known whether there is any general property of the orbits that is conserved during a collision, something which corresponds, for example, to the conservation of energy for collisions between the molecules of a gas. With this idea in mind, the mechanics of an interaction in which $d$, the minimum distance of separation, is always much greater than $a$, a typical dimension of the particles, was worked out. Since if there is a quantity that is conserved for a general interaction, it will be conserved for an interaction in which $a / d$ is small, and it is possible to carry out the analysis for this case by neglecting powers of $a / d$ higher than the third.

The result of this lengthy calculation was that the arbitrary parameters that give the relative configuration of the particles before the interaction commences can be so varied that the orbits of the particles can be altered independently by the interaction. Further, it was found that the orbits of the particles are changed by the interaction in such a way that the statistical properties of the suspension are unaltered. Hence, there is no conservation principle, so that the ideas of Statistical Mechanics cannot be applied, and it is necessary to consider the detailed mechanics of a close interaction or collision before information about the effect of collisions can be obtained; unfortunately, there appears to be no way of doing this.

Finally, there are two intuitive hypotheses about the effect of collisions that are perhaps worth mentioning. The first of these is that those orbits are favoured for which the energy dissipation is small, and the second is that the orbits favoured are those for which the volume swept out by the particle is small. The first hypothesis is reminiscent of Jeffery's, but the situation here is different from that of $\S 4$ since we are concerned with solutions of equations (1) and not with the effect of terms neglected in these equations. The second one, which was suggested to me by Dr J. W. Glen, is based on the intuitive idea that the smaller the volume swept out by a particle, the smaller the frequency of collisions made by the particle, with the result that the particle stays in its orbit longer. It follows from the first hypothesis that there is a tendency for particles to align themselves with the long axis along the direction of the vortex lines, so that $p(k)$ is large for large $k$ if the particles are prolate and for small $k$ if they are oblate; and it follows from the second hypothesis that the tendency is for the axis of symmetry to lie along the direction of the vortex lines, so that $p(k)$ is large for large $k$ for both prolate and oblate particles. Thus, for prolate particles, the predictions are the same and in accordance with the observations of Manley \& Mason 
(1956), but the predictions for oblate particles are in disagreement. An experiment using oblate particles would be of some interest in this connection.

I wish to thank Sir Geoffrey Taylor for suggesting this problem to me, and I am grateful to both him and Dr G. K. Batchelor for the kind interest that they have shown in this work. I also wish to thank Dr K. Weissenberg for the supply of some ammonium polymethyl acrilate.

\section{REFERENCES}

Binder, A. 1939 F. Appl. Phys. 10, 711.

Jeffery, G. B. 1922 Proc. Roy. Soc. A, 102, 161.

LoREntz, H. A. 1907 Abhandlungen uber Theoretische Physik, p. 23. Leipzig.

Manley, R. St. J., ARlov, A. P. \& Mason, S. G. 1955 Nature, Lond. 175, 682.

Manley, R. St. J. \& Mason, S. G. 1956 To be published.

TAYLOR, G. I. 1923 Proc. Roy. Soc. A, 103, 58.

Tolman, R. C. 1938 The Principles of Statistical Mechanics. Oxford University Press.

Trevelyan, B. J. \& Mason, S. G. 1951 F. Coll. Sci. 6, 354.

Trubsdel. C. 1952 F. Rat. Mech. Anal. 1, 125.

WeIsSEnBERg, K. 1947 Nature, Lond. 159, 310.

Whitehead, A. N. 1893 Q:tart. F. Math. 23, 143. 